\title{
Risk Profiling of Cancer Treatment-Related Cardiovascular Disorders in Breast Cancer Patients Who Received Adjuvant Chemotherapy With Trastuzumab
}

\author{
Hiromi Shinano; Sakiko Miyazaki, MD, PhD; Kayo Miura, MD, PhD; \\ Hiroshi Ohtsu; Naohiro Yonemoto; Kiyoshi Matsuoka; Hakuou Konishi, MD, PhD; \\ Hiroyuki Daida, MD, PhD; Mitsue Saito, MD, PhD; Kazuhiro Sase, MD, PhD
}

\begin{abstract}
Background: The prognosis of cancer survivors has dramatically improved, but effective strategies for cancer treatment-related cardiovascular disorders (CTRCD) remain to be elucidated in the emerging field of cardio-oncology. In this study, we investigated risk factors for CTRCD in breast cancer patients treated with trastuzumab.

Methods and Results: We performed a retrospective analysis of 141 consecutive women who received adjuvant trastuzumab, and underwent baseline (BL) and follow-up (FU) echocardiography at Juntendo University between April 2010 and December 2016. The major concomitant treatment was anthracyclines in $94 \%$ and radiotherapy in $53 \%$. During the median treatment period of 11 months, there were $22(15.6 \%)$ cardiology consultations, $3(2.1 \%)$ treatment interruptions with irreversible CTRCD, and no deaths. Left ventricular ejection fraction (LVEF) was decreased from a median $67.5 \%$ (BL) to $63.4 \%$ (FU; $P<0.0001$ ), with reduced LVEF noted in $26.2 \%$ at $\mathrm{FU}<90 \% \mathrm{BL}$, in $13.5 \%$ at $\mathrm{FU}<\mathrm{BL}-10 \%$, and in $5.7 \%$ at $\mathrm{LVEF} F \mathrm{~F}<5 \%$. A significantly greater percentage of patients with CTRCD (FU<BL-10\% and LVEFFU $53 \%$ ) had cardiovascular risk factors (CVRF; $42.9 \%$ vs. 8.2\%, $\mathrm{P}=0.02$ ). On multivariable analysis, CVRF were also significantly associated with CTRCD (OR, 11.96; 95\% Cl: 1.30-110.34).
\end{abstract}

Conclusions: Adjuvant trastuzumab for early-stage breast cancer was associated with reduced LVEF; and CVRF were an independent predictor for CTRCD. The concomitant effect of anthracyclines should not be underestimated, even at lower doses.

Key Words: Breast cancer; Cancer treatment-related cardiovascular disorder; Cardio-oncology; Cardiovascular risk factor; Trastuzumab

$\mathbf{P}$ rogress in medical care for cancer has greatly contributed to lower mortality rates in affected patients, resulting in the increasing importance of cancer survivorship care in Japan ${ }^{1}$ and throughout the world. ${ }^{2}$ Epidemiological studies indicate that one of the most important causes of death in cancer survivors is cardiovascular disease (CVD). ${ }^{3}$ In addition to common risk factors for CVD and cancer, ${ }^{4}$ there is also growing awareness of cancer treatment-related cardiovascular disorders (CTRCD) associated with traditional ${ }^{5}$ and modern treatment strategies. ${ }^{68}$

Cardio-oncology is a rapidly developing field in which cardiologists and oncologists collaborate to deal with novel issues including CTRCD, with a common goal of improving the prognosis of cancer patients. ${ }^{9}$ The compelling clinical need for cardio-oncology services was initially recognized by major educational hospitals, ${ }^{\mathbf{1 0}}$ then those efforts gradually developed into national ${ }^{11,12}$ and academic ${ }^{13}$ collaborations to conduct research, resulting in publication of important findings and evidence-based clinical practice guidelines. ${ }^{14-16}$

Trastuzumab, one of the first available molecular targeted therapies, has been found to significantly improve the prognosis of human epidermal growth factor receptor-2

Received November 14, 2019; revised manuscript received January 31, 2020; accepted February 18, 2020; J-STAGE Advance Publication released online March 24, 2020 Time for primary review: 24 days

Clinical Pharmacology and Regulatory Science (H.S., K.S.), Department of Cardiovascular Medicine (S.M., H.K., H.D.), Department of Breast and Endocrine Surgery (K. Miura, M.S.), Graduate School of Medicine, Juntendo University, Tokyo; Leading Center for the Development and Research of Cancer Medicine (H.O.), Innovative Medical Technology Research \& Development Center (N.Y., K. Matsuoka, H.D.), Juntendo University, Tokyo; Center for Clinical Sciences, Department of Data Science, National Center for Global Health and Medicine, Tokyo (H.O., K. Matsuoka); Department of Neuropsychopharmacology, National Institute of Mental Health, National Center of Neurology and Psychiatry, Tokyo (N.Y.); and Institute for Medical Regulatory Science, Organization for University Research Initiatives, Waseda University, Tokyo (H.O., K.S.), Japan

H.D. is a member of Circulation Reports' Editorial Team.

Mailing address: Kazuhiro Sase, MD, PhD, Clinical Pharmacology and Regulatory Science, Juntendo University Graduate School of Medicine, 2-1-1 Hongo, Bunkyo-ku, Tokyo 113-8421, Japan. E-mail: sase@juntendo.ac.jp

All rights are reserved to the Japanese Circulation Society. For permissions, please e-mail: cr@j-circ.or.jp

ISSN-2434-0790 


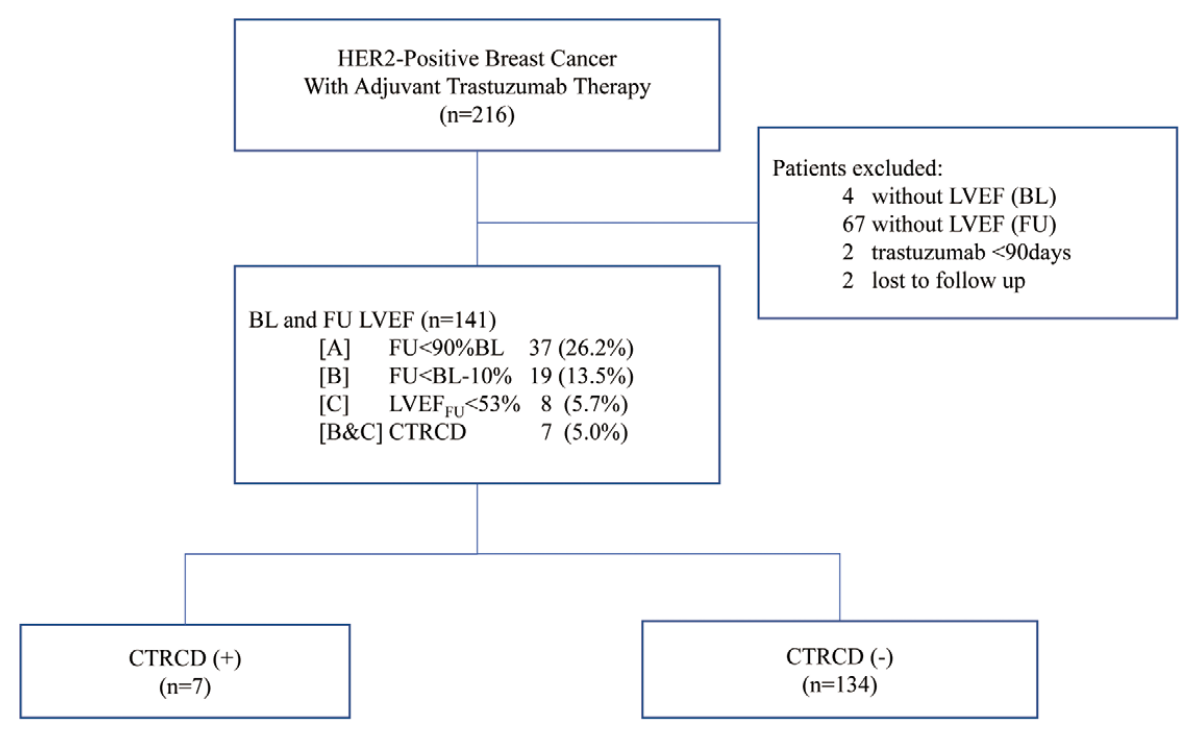

Figure 1. Subject selection. CTRCD, cancer therapy-related cardiovascular disorders; BL, baseline; FU, follow-up; HER2, human epidermal growth factor receptor-2; LVEF, left ventricular ejection fraction.

(HER2)-positive breast cancer. ${ }^{17-19}$ It was initially approved for the treatment of metastatic disease, ${ }^{17}$ and subsequently for adjuvant chemotherapy for early-stage breast cancer. ${ }^{18}$ Cardiotoxicity was also identified in clinical trials, especially in patients receiving concurrent anthracyclines, and a warning has been included prominently on the label since approval was obtained for use. ${ }^{20}$ The process of developing cardio-oncology guidelines highlighted the lack of uniformity of cardiac assessment during trastuzumab therapy. ${ }^{21}$ For example, some oncology guidelines that distinguish trastuzumab from anthracycline cardiotoxicity do not recommend excessive monitoring to avoid unnecessary discontinuation of cancer treatment. ${ }^{22}$ In contrast, most cardiology guidelines recommend routine monitoring of biomarkers and imaging to detect asymptomatic cardiac dysfunction rather than waiting for symptomatic heart failure onset. ${ }^{23}$ In other words, insufficient data are available to identify high-risk patients as well as high-risk treatments for CTRCD in this new field of interdisciplinary research, demonstrating an urgent need for clinical evidence with which to develop risk $\operatorname{scores}^{\mathbf{2 4}}$ and validate surrogate markers. ${ }^{25}$

The aim of the present study was therefore to provide real-world data from a cardio-oncology viewpoint regarding risk profiling and the cardiac assessment of patients with breast cancer in Japan who are receiving adjuvant trastuzumab.

\section{Methods}

\section{Subjects}

The study flow chart is shown in Figure 1. A total of 216 consecutive female patients with HER2-positive breast cancer undergoing adjuvant trastuzumab therapy at Juntendo University Hospital (Tokyo, Japan) between April 2010 and December 2016 were retrospectively identified from hospital records. Those without baseline (BL) echocardiography $(n=4)$, without follow-up $(F U)$ for left ventricular ejection fraction ( $\mathrm{LVEF} ; \mathrm{n}=67$ ), with $<90$ days of trastuzumab therapy $(n=2)$, and who were lost to FU $(n=2)$ were excluded from analysis.

\section{Data Collection}

BL information collected from the medical records included age, body mass index (BMI), cardiovascular risk factors (CVRF; i.e., hypertension, diabetes, dyslipidemia, CVD), and details regarding cancer treatment (i.e., concurrent chemotherapy regimens, surgery, radiation therapy).

The protocol for this retrospective study was designed in accordance with the Declaration of Helsinki and the Ethics Guidelines for Clinical Research of the Ministry of Health, Labour, and Welfare of Japan, and received approval from the Juntendo University Hospital Ethics Committee (17271). Informed consent was not required because this was an observational study with an opportunity to opt out. All patient data were anonymized prior to statistical analysis.

\section{Cardiac Assessment}

LVEF measurements (modified Simpson's method) were performed at BL (minimum LVEF before trastuzumab therapy) and at FU (minimum LVEF after trastuzumab treatment).

\section{Dysfunction and CTRCD}

Of several proposed criteria for LV dysfunction, ${ }^{26} 3$ thresholds for LVEF were evaluated in the present study as follows: A, 10\% relative decrease from BL (FU<90\% BL); $\mathrm{B}$, absolute decrease of $10 \%$ from $\mathrm{BL}(\mathrm{FU}<\mathrm{BL}-10 \%)$; and $\mathrm{C}$, decrease to below institutional normal ( $\mathrm{LVEFFU}_{\mathrm{F}}<53 \%$ ). For the present analyses, CTRCD was defined according to a previous consensus statement (B and C). ${ }^{23}$

\section{Statistical Analysis}

Continuous variables are expressed as mean \pm SD or median (IQR), and were compared using Student's t-test or Wilcoxon rank sum test. Categorical variables are expressed 
as percentage or frequency, and were compared using Fisher's exact test or chi-squared test. Logistic regression analysis was used to compare odds ratios (OR) and 95\% CI. Age, anthracycline use, radiotherapy, and BMI were selected as covariates for multivariate analysis. Probability values are 2 -tailed and $\mathrm{P}<0.05$ was considered to indicate statistical significance. All analyses were performed using SAS ver. 9.4 (SAS Institute, Cary, NC, USA).

\section{Results}

\section{Subjects}

The present analysis involved 141 female patients with HER2-positive breast cancer who underwent adjuvant trastuzumab therapy. Their BL clinical features are listed in Table 1. Mean age was 54 years and the median duration of trastuzumab therapy was 11.0 months (IQR, 10.7-13.3 months). The majority $(94.3 \%)$ of these patients received anthracycline chemotherapy in addition to trastuzumab, while approximately half $(53.2 \%)$ underwent radiation therapy and were analyzed according to the site of irradiation.

\section{Cardiac Assessment and Clinical Outcome}

LVEF was significantly lower at FU as compared with BL (63.4\%; IQR, 58.2-68.5\% vs. $67.5 \%$, IQR, $64.4-70.5 \%$, $\mathrm{P}<0.0001$; Figure 2). Detailed analysis of the threshold for LV dysfunction is shown in Figure 3. The numbers of CTRCD patients with A, 10\% relative LVEF decrease from $\mathrm{BL}(\mathrm{FU}<90 \% \mathrm{BL})$; B, absolute LVEF decrease of $10 \%$ from BL $(\mathrm{FU}<\mathrm{BL}-10 \%)$; and $\mathrm{C}$, LVEF decrease to below institutional normal (LVEFFu $<53 \%$ ); and that of $\mathrm{B}$ and C combined were $37(26.2 \%), 19(13.5 \%), 8(5.7 \%)$, and $7(5.0 \%)$, respectively. Twenty-two patients $(15.6 \%)$ underwent consultation with a cardiologist; and discontinuation of trastuzumab treatment due to CTRCD was noted in 3 $(2.1 \%)$. During the FU period, none of the patients died of a tumor-related cause and no cardiac deaths were observed.

\section{CTRCD Risk Profiling}

Patients were subdivided into $\operatorname{CTRCD}(+)(\mathrm{n}=7)$ and CTRCD $(-)(n=134)$ groups (Table 2). There were no differences in regard to duration of trastuzumab or of combination therapy, or BMI between those groups. In contrast, there was a significant difference in regard to age between patients with and without CTRCD $(63.0 \pm 10.7$ vs. $53.5 \pm 11.4$ years, $\mathrm{P}=0.04)$, and with and without $\mathrm{CVRF}$ ( $42.9 \%$ vs. $8.2 \%, \mathrm{P}=0.02)$. On multivariate analysis, $\mathrm{CVRF}$ at $\mathrm{BL}$ independently predicted subsequent development of CTRCD (OR, 11.96; 95\% CI: 1.30-110.3; Table 3). The relationship between LVEF change (BL-FU) and CVRF is shown in Figure 4.

\section{Discussion}

In this retrospective observational study of 141 consecutive female patients with early-stage breast cancer who underwent trastuzumab therapy in Japan, we found 3 cases $(2.1 \%)$ of cancer treatment discontinuation in 7 patients $(5.0 \%)$ who developed CTRCD. The incidence of CTRCD, defined as LVEF FU $<\mathrm{BL}-10 \%$ and $\mathrm{LVEF}_{\mathrm{FU}}<53 \%$, was similar to previous reports. ${ }^{26} \mathrm{CVRF}$ (hypertension, diabetes, dyslipidemia, or history of heart disease) as well as age have been identified as predictors of CTRCD. The effects of anthracyclines, even in lower doses, have not been ruled

\begin{tabular}{|c|c|}
\hline & $\begin{array}{l}\text { Overall } \\
(n=141)\end{array}$ \\
\hline \multicolumn{2}{|l|}{ Patient demographics } \\
\hline Age (years) & $54.0 \pm 11.5$ \\
\hline Age $>65$ & $27(19.2)$ \\
\hline $\operatorname{BMI}\left(\mathrm{kg} / \mathrm{m}^{2}\right)$ & $21.9 \pm 3.3$ \\
\hline Underweight $(\leq 18.4)$ & $22(15.6)$ \\
\hline Normal (18.5-24.9) & $90(63.8)$ \\
\hline Overweight (25.0-29.9) & $27(19.2)$ \\
\hline Obese $(\geq 30.0)$ & $2(1.4)$ \\
\hline CVRF & $14(9.9)$ \\
\hline Hypertension & $6(4.3)$ \\
\hline Diabetes mellitus & $3(2.1)$ \\
\hline Hypercholesterolemia & $10(7.1)$ \\
\hline Cardiac disease & $1(0.7)$ \\
\hline \multicolumn{2}{|l|}{ Breast cancer treatment } \\
\hline Trastuzumab treatment (months) & $11.0(10.7-13.3)$ \\
\hline Adjuvant chemotherapy & $74(52.5)$ \\
\hline Neoadjuvant chemotherapy & $67(47.5)$ \\
\hline Anthracyclines & $133(94.3)$ \\
\hline Epirubicin $\left(\mathrm{mg} / \mathrm{m}^{2}\right)$ & $400.0(266.1-412.6)$ \\
\hline Cyclophosphamide & $128(90.8)$ \\
\hline $5-\mathrm{FU}$ & $128(90.8)$ \\
\hline Taxanes & $80(56.7)$ \\
\hline Endocrine treatment & $5(3.6)$ \\
\hline RT (All) & 75 (53.2) \\
\hline Dose (Gy) & $44.0(41.0-49.8)$ \\
\hline Left Breast & $41(29.1)$ \\
\hline Dose (Gy) & $44.0(40.7-49.6)$ \\
\hline Right Breast & $36(25.5)$ \\
\hline Dose (Gy) & $47.0(40.5-49.8)$ \\
\hline
\end{tabular}

Data given as $\mathrm{n}(\%)$, mean \pm SD or median (IQR). Endocrine treatment includes aromatase inhibitors (anastrozole, letrozole), selective estrogen receptor modulator (tamoxifen), and $\mathrm{GnRH}$ agonist (goserelin). 5-FU, 5-fluorouracil; BMI, body mass index; CVRF, cardiovascular risk factors; RT, radiotherapy.

out. The present findings show that even in Japan or Asia, where the incidence of heart disease is considered to be lower than that in the USA and Europe, ${ }^{27-29}$ there is a significant number of types of asymptomatic LV dysfunction as well as a small but important number of types of symptomatic heart failure, which can potentially worsen the prognosis of cancer survivors. ${ }^{30,31}$

The present results are consistent with recent trends in cardio-oncology research. Immediately after the introduction of molecular targeted drugs, cardiotoxicity was classified into type I (irreversible) and type II (reversible), ${ }^{6}$ although increasing evidence suggests that this classification is incomplete and inaccurate. ${ }^{32}$ First, because heart failure is a progressive condition from stage A (risk), to stage B (asymptomatic) and then stage $\mathrm{C} / \mathrm{D}$ (symptomatic), it cannot be classified by the type of drug treatment alone. ${ }^{33}$ Second, although trastuzumab toxicity has been shown to be the second most common type of cardiotoxicity, the classification of cardiotoxicity can no longer be limited to 2 types following the introduction of a wide variety of cancer treatments. ${ }^{34}$ Thus, in clinical practice, trastuzumab cardiotoxicity should not be considered reversible. ${ }^{35}$ Instead, 


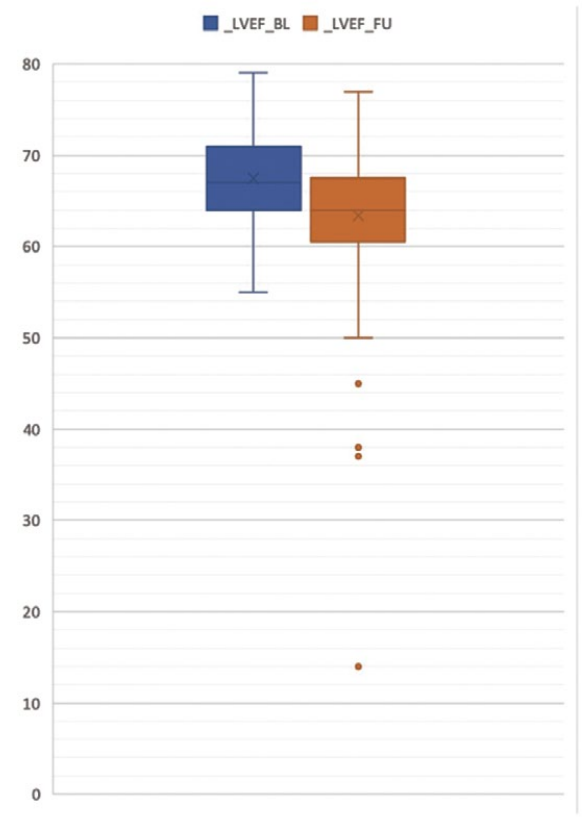

\begin{tabular}{lcccccccc}
\hline LVEF (\%) & \multicolumn{2}{c}{ Baseline (BL) } & \multicolumn{2}{l}{ Follow-up (FU) } & \multicolumn{2}{l}{ BL-FU } & $P$-value \\
\hline$(\mathrm{n}=141)$ & 67.5 & {$[64.4-70.5]$} & 63.4 & {$[58.2-68.5]$} & 4.1 & {$[-1.1-9.4]$} & $<0.0001$ \\
\hline
\end{tabular}

Figure 2. Cardiac assessment. Data given as median (IQR). BL, baseline; FU, follow-up; LVEF, left ventricular ejection fraction (\%).

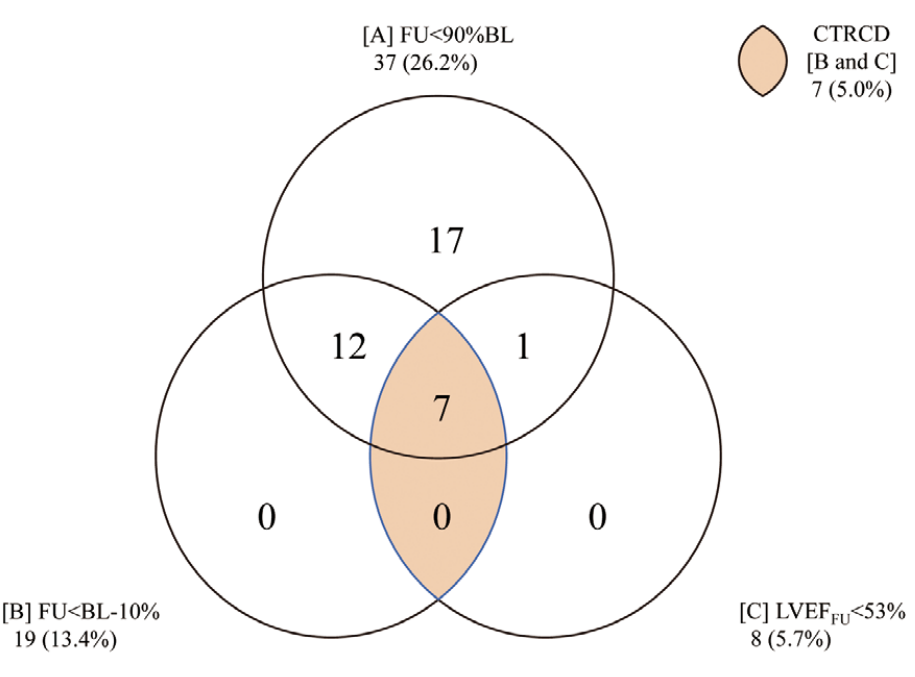

Figure 3. Thresholds of left ventricular ejection fraction (LVEF) and definition of cancer treatmentrelated cardiovascular disorders (CTRCD). BL, baseline; FU, follow-up.

it is important to identify high-risk patients and high-risk treatments before initiation of cancer treatment, followed by monitoring during treatment and during FU sessions after treatment, depending on the individual risk status. ${ }^{\mathbf{1 6}}$

Significant progress has been made in breast cancer treatment options, such as trastuzumab therapy, and the importance of cardiologists in a team-based approach has been noted. ${ }^{36}$ CVD is the second most common cause of death and in some subgroups, such as postmenopausal women with hormone receptor positive $(\mathrm{HR}+)$ breast cancer, death from CVD is more common than from cancer. ${ }^{4}$ Additionally, there are common risk factors shared by breast cancer and CVD, such as smoking, family history, and obesity. ${ }^{37}$ The present results indicate the importance of CVRF in addition to chemotherapy, molecular and endocrine therapy, and radiation therapy for patients with breast cancer. Prevention and management of CVD by cardiologists is expected to support completion of optimal cancer treatment, thus improving overall survival with the use of a team-based approach. ${ }^{16}$ 


\begin{tabular}{|c|c|c|c|}
\hline CTRCD & $(+)(n=7)$ & $(-)(n=134)$ & P-value \\
\hline \multicolumn{4}{|l|}{ Patient demographics } \\
\hline Age (years) & $63.0 \pm 10.7$ & $53.5 \pm 11.4$ & 0.04 \\
\hline Age $>65$ & $4(57.1)$ & $23(17.2)$ & \\
\hline $\operatorname{BMI}\left(\mathrm{kg} / \mathrm{m}^{2}\right)$ & $20 \pm 2.0$ & $22 \pm 3.3$ & 0.13 \\
\hline Underweight $(\leq 18.4)$ & $2(28.6)$ & $20(14.9)$ & \\
\hline Normal (18.5-24.9) & $5(71.4)$ & $85(63.4)$ & \\
\hline Overweight (25.0-29.9) & $0(0.0)$ & $27(20.2)$ & \\
\hline Obese $(\geq 30.0)$ & $0(0.0)$ & $2(1.5)$ & \\
\hline CVRF & $3(42.9)$ & $11(8.2)$ & 0.02 \\
\hline Hypertension & $3(42.9)$ & $5(3.7)$ & \\
\hline Diabetes mellitus & $0(0.0)$ & $3(2.2)$ & \\
\hline Hypercholesterolemia & $1(14.3)$ & $9(6.7)$ & \\
\hline Cardiac disease & $1(14.3)$ & $0(0.0)$ & \\
\hline \multicolumn{4}{|l|}{ Breast cancer treatment } \\
\hline Trastuzumab (months) & $11(7.8-13.1)$ & $11(10.9-13.3)$ & 0.54 \\
\hline Adjuvant chemotherapy & $3(42.9)$ & $71(53.0)$ & \\
\hline Neoadjuvant chemotherapy & $4(57.1)$ & $63(47.0)$ & \\
\hline Anthracyclines & $7(100.0)$ & $126(94.0)$ & \\
\hline Epirubicin $\left(\mathrm{mg} / \mathrm{m}^{2}\right)$ & $300(295.7-361.5)$ & $400(265.1-414.7)$ & 0.27 \\
\hline Cyclophosphamide & $7(100.0)$ & $121(90.3)$ & \\
\hline $5-\mathrm{FU}$ & $7(100.0)$ & $121(90.3)$ & \\
\hline Taxanes & $5(71.4)$ & $75(56.0)$ & \\
\hline Endocrine treatment & $0(0.0)$ & $5(3.7)$ & \\
\hline RT & $3(42.9)$ & $72(53.7)$ & 0.71 \\
\hline Dose (Gy) & $46.6(43.4-49.8)$ & $44.0(40.9-49.8)$ & \\
\hline Left Breast & $2(28.6)$ & $39(29.1)$ & 1.00 \\
\hline Dose (Gy) & $50.0(50.0-50.0)$ & $44.0(43.2-50.0)$ & \\
\hline Right Breast & $1(14.3)$ & $35(26.1)$ & 1.00 \\
\hline Dose (Gy) & $43.2(43.2-43.2)$ & $48.0(43.2-50.0)$ & \\
\hline \multicolumn{4}{|l|}{ Cardiovascular outcomes } \\
\hline No. LVEF evaluations & $8(5.5-8.5)$ & $4(3.1-5.3)$ & \\
\hline Cardiologist consultation & $4(57.1)$ & $18(13.4)$ & \\
\hline Discontinuation & $3(2.1)$ & & \\
\hline
\end{tabular}

Data given as $\mathrm{n}(\%)$, mean \pm SD or median (IQR). Endocrine treatment includes aromatase inhibitors (anastrozole, letrozole), selective estrogen receptor modulator (tamoxifen), and GnRH agonist (goserelin). CTRCD, cancer treatmentrelated cardiovascular disorders; LVEF, left ventricular ejection fraction. Other abbreviations as in Table 1.

Although trastuzumab was one of the first molecular targeted treatments approved for cancer treatment, the mechanism related to its cardiotoxicity is not well understood. ${ }^{38}$ The drug is a monoclonal antibody that binds to HER2 and decreases its signaling. Intensive benefit-risk analyses of trastuzumab have been performed in basic as well as clinical research studies. Addition of trastuzumab to standard chemotherapy has been shown to improve the outcomes of metastatic as well as of early-stage HER2positive breast cancer. ${ }^{17,18}$ Especially, for patients with early-stage disease in whom the goal is cure, systemic chemotherapy given before (neoadjuvant) or after (adjuvant) surgery is critically important to eradicate micrometastatic disease and decrease the risk of recurrence. ${ }^{36} \mathrm{In}$ our experience, the clinically most important side-effect preventing completion of trastuzumab treatment for earlystage breast cancer is cardiotoxicity, which results in various conditions from asymptomatic myocardial damage to development of symptomatic heart failure. Neuregulin-1 (NRG-1) is an essential protein in adult myocardium tissues

\begin{tabular}{|lcc|}
\hline \multicolumn{3}{|c|}{ Table 3. Multivariate Indicators of CTRCD } \\
CVRF & OR & 95\% Cl \\
Age (years) & 11.968 & $1.298-110.34$ \\
Dose (anthracycline) & 1.054 & $0.971-1.145$ \\
Radiotherapy & 1.001 & $0.990-1.012$ \\
BMI & 0.519 & $0.084-3.230$ \\
\hline
\end{tabular}

Abbreviations as in Tables 1,2.

for cell cycle survival, because it promotes sarcomere stability and reduces oxidative stress. HER 2 binds with NRG-1 to maintain cell survival and stabilizes signals that prevent apoptosis in response to stress, while trastuzumab is considered to block these pathways by inhibiting the HER2 receptor. ${ }^{38}$ Further elucidation of the pathophysiological factors is necessary for prevention, diagnosis, and treatment of trastuzumab cardiotoxicity. ${ }^{39}$ 


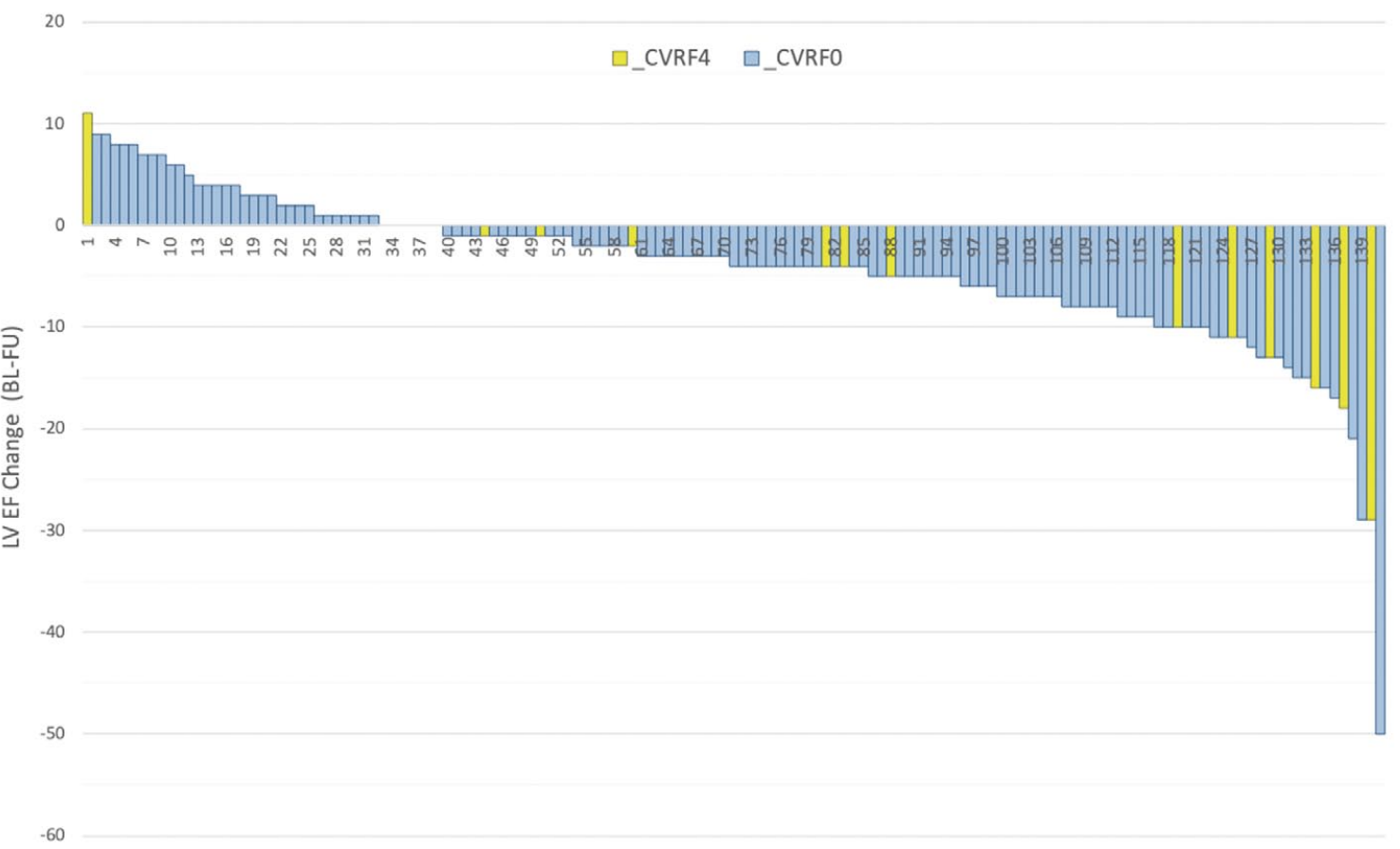

Figure 4. Left ventricular ejection fraction (LVEF) change (baseline-follow-up [BL-FU]) and cardiovascular risk factors (CVRF). CVRFO, patient without CVRF; CVRF4, patient with $\geq 1$ of 4 CVRF.

Information regarding CTRCD has been available for some time. For example, anthracyclines have been known to be associated with severe and irreversible heart failure since the $1970 \mathrm{~s}$, and attention must be given to cumulative dose over the lifetime of a treated patient. ${ }^{40}$ Although initially thought to be due to oxidative stress, pathological conditions at the molecular cell level have been clarified in recent years, although additional research as well as development of cardioprotective drugs are needed.41 Radiation therapy, especially for left breast cancer with cardiac radiation, is known to be associated with cardiovascular adverse events, while recent technological advancements have reduced the incidence of radiation-induced CVD. ${ }^{\mathbf{4 2}}$ Additionally, new therapies, such as molecular targeted drugs and immune checkpoint inhibitors, have been approved, with CTRCD, a completely new pathology, also emerging. ${ }^{43}$ For example, vascular endothelial growth factor inhibitors such as bevacizumab for colorectal cancer, and proteasome inhibitors such as bortezomib for multiple myeloma, as well as immune checkpoint inhibitors such as nivolumab for malignant melanoma and pembrolizumab for lung cancer have been shown to be effective. ${ }^{34}$ However, many new CTRCD for the aforementioned conditions, such as cardiac dysfunction, coronary artery disease, peripheral artery disease, arrhythmia, hypertension, valvular disease, and venous thromboembolism, have never been encountered by the majority of cardiologists. ${ }^{44}$ Therefore, there is a compelling need for a new interdisciplinary area of cardio-oncology, in which oncologists and cardiologists collaborate for the better understanding of treatment options.

Cardio-oncology as well as onco-cardiology represent a patient-centered collaboration between cardiologists and oncologists with the common goal of maximizing the outcome of cancer treatment while minimizing the risk of CVD. ${ }^{45}$ Each started as a team-based approach by healthcare providers to dealing with cardiotoxicity related to anthracycline or radiation therapy. ${ }^{10}$ Subsequently, the necessity of basic, clinical, and epidemiological research has been examined as a response at both national and academic levels. ${ }^{46}$ These public-private partnerships have led to a dramatic increase in competitive research funds and published papers. Presently, discussions are underway to build a sustainable system, involving the creation of clinical guidelines and input regarding medical insurance reimbursement. ${ }^{11,12}$

Cardiac assessment during and after cancer treatment is expected to improve prevention, diagnosis, and treatment of symptomatic CTRCD, and also help to avoid discontinuation of cancer treatment. Although the present retrospective observational study suggests that monitoring $\mathrm{LVEF}$, as specified in the trastuzumab package insert, is important for diagnosis of CTRCD, it may not be sufficient for prevention. ${ }^{39}$ Currently, there are ongoing investigations to quantify LVEF for early detection of CTRCD using a variety of techniques, ${ }^{25}$ including 2-D and 3-D echocardiography, global longitudinal strain, ${ }^{47}$ tissue Doppler imaging, multigated acquisition scanning, and cardiac magnetic resonance imaging. Additionally, biomarkers including B-type natriuretic peptide, cardiac troponins, and others are also being studied. ${ }^{48}$ From a cardio-oncology perspective, there is urgent need to validate surrogate endpoints for both positive and negative predictive values based on sensitivity, specificity, and cut-offs, in order to encourage early detection of CTRCD while avoiding premature termination of therapy due to overdiagnosis.11,12 


\section{Study Limitations}

There were some limitations associated with this retrospective, single-center observational study conducted at a Japanese educational hospital. First, there are potential confounding factors, biases, and missing values. For example, not all of the pre-treatment CVRF have been adjudicated by cardiologists. In addition, the study design did not have sufficient statistical power to rule out the influence of concomitant therapies including irradiation site and anthracycline therapy. Therefore, causal relationships between treatment strategy, CVRF, and CTRCD must be carefully interpreted. Second, attention should be paid to the characteristics of the hospital. Of the 216 patients screened in this study, only 4 did not have BL LVEF measurements available. However, real-world data show gaps between guidelines and actual clinical practice. ${ }^{21}$ Therefore, additional investigations are needed regarding the incidence and prognosis of CTRCD in situations in which cardiovascular monitoring is less frequently used. Third, although we used LVEF to evaluate CTRCD in this study, there are known issues, including sensitivity, specificity, and reproducibility, related to the use of 2-D echocardiography as a tool to detect CTRCD. ${ }^{\mathbf{2 6}}$ Nevertheless, it is important to note that there are ongoing investigations to validate various imaging modalities as well as examinations of biomarkers before, during, and after highrisk treatment for high-risk patients in order to minimize CTRCD and maximize the outcome of cancer therapies. ${ }^{16}$ Finally, with regard to ethnicity factors, heart disease is known to have a lower prevalence in Japan and in Asia as compared with Western populations due to both genetic and environmental factors, although it is showing an increasing trend along with economic development. A future investigation is needed to determine whether a similar trend exists for CTRCD. ${ }^{29}$

\section{Conclusions}

Although this retrospective analysis indicates an increased prevalence of CTRCD in patients with potentially modifiable CVRF who underwent adjuvant trastuzumab therapy, there are multiple factors to be considered before, during, and after cancer treatment for prevention, diagnosis, and treatment of cardiovascular comorbidities. Development of CTRCD disrupts the risk-benefit balance for patients with early-stage cancer, emphasizing the need for additional studies to identify at-risk populations, validate monitoring strategies, and establish evidence-based clinical practice guidelines for the field of cardio-oncology.

\section{Acknowledgments}

We are grateful for the excellent support from the staff at Juntendo University Hospital Breast Center, Heart Center, and Medical Record Management Office.

This study was supported in part by a JSPS/MEXT Grant-in-Aid for Scientific Research $(18 \mathrm{~K} 12134$, K.S.) and a grant from the National Center for Global Health and Medicine (29-S-1004, H.O.).

\section{Disclosures}

The authors declare no conflicts of interest. H.D. is a member of Circulation Reports' Editorial Team.

\section{References}

1. Katanoda K, Hori M, Matsuda T, Shibata A, Nishino Y, Hattori $\mathrm{M}$, et al. An updated report on the trends in cancer incidence and mortality in Japan, 1958-2013. Jpn J Clin Oncol 2015; 45: 390-
401.

2. Shapiro CL. Cancer survivorship. $N$ Engl J Med 2018; 379: $2438-2450$

3. Armenian SH, Xu L, Ky B, Sun C, Farol LT, Pal SK, et al. Cardiovascular disease among survivors of adult-onset cancer: A community-based retrospective cohort study. J Clin Oncol 2016; 34: $1122-1130$.

4. Mehta LS, Watson KE, Barac A, Beckie TM, Bittner V, CruzFlores S, et al. Cardiovascular disease and breast cancer: Where these entities intersect: A scientific statement from the American Heart Association. Circulation 2018; 137: e30-e66.

5. Cardinale D, Colombo A, Lamantia G, Colombo N, Civelli M, De Giacomi G, et al. Anthracycline-induced cardiomyopathy: Clinical relevance and response to pharmacologic therapy. $J \mathrm{Am}$ Coll Cardiol 2010; 55: 213-220.

6. Ewer MS, Ewer SM. Cardiotoxicity of anticancer treatments: What the cardiologist needs to know. Nat Rev Cardiol 2010; 7: $564-575$.

7. Minami M, Matsumoto S, Horiuchi H. Cardiovascular side-effects of modern cancer therapy. Circ J 2010; 74: 1779-1786.

8. Moslehi JJ. Cardiovascular toxic effects of targeted cancer therapies. N Engl J Med 2016; 375: 1457-1467.

9. Lenihan DJ, Cardinale D, Cipolla CM. The compelling need for a cardiology and oncology partnership and the birth of the International CardiOncology Society. Prog Cardiovasc Dis 2010; 53: $88-93$.

10. Snipelisky D, Park JY, Lerman A, Mulvagh S, Lin G, Pereira N, et al. How to develop a cardio-oncology clinic. Heart Fail Clin 2017; 13: 347-359.

11. Shelburne N, Adhikari B, Brell J, Davis M, Desvigne-Nickens P, Freedman A, et al. Cancer treatment-related cardiotoxicity: Current state of knowledge and future research priorities. J Natl Cancer Inst 2014; 106: dju232.

12. Shelburne N, Simonds NI, Adhikari B, Alley M, Desvigne-Nickens $\mathrm{P}$, Dimond $\mathrm{E}$, et al. Changing hearts and minds: Improving outcomes in cancer treatment-related cardiotoxicity. Curr Oncol Rep 2019; 21: 9.

13. Barac A, Murtagh G, Carver JR, Chen MH, Freeman AM, Herrmann J, et al. Cardiovascular health of patients with cancer and cancer survivors: A roadmap to the next level. $J$ Am Coll Cardiol 2015; 65: 2739-2746.

14. Armenian SH, Hudson MM, Mulder RL, Chen MH, Constine LS, Dwyer M, et al. Recommendations for cardiomyopathy surveillance for survivors of childhood cancer: A report from the International Late Effects of Childhood Cancer Guideline Harmonization Group. Lancet Oncol 2015; 16: e123-e136.

15. Zamorano JL, Lancellotti P, Rodriguez Munoz D, Aboyans V, Asteggiano R, Galderisi M, et al. 2016 ESC Position Paper on cancer treatments and cardiovascular toxicity developed under the auspices of the ESC Committee for Practice Guidelines: The Task Force for cancer treatments and cardiovascular toxicity of the European Society of Cardiology (ESC). Eur Heart J 2016; 37: $2768-2801$.

16. Armenian SH, Lacchetti C, Barac A, Carver J, Constine LS, Denduluri N, et al. Prevention and monitoring of cardiac dysfunction in survivors of adult cancers: American Society of Clinical Oncology Clinical Practice Guideline. J Clin Oncol 2017; 35: $893-911$.

17. Slamon D, Leyland-Jones B, Shak S, Fuchs H, Paton V, Bajamonde $\mathrm{A}$, et al. Use of chemotherapy plus a monoclonal antibody against HER2 for metastatic breast cancer that overexpresses HER2. N Engl J Med 2001; 344: 783-792.

18. Slamon D, Eiermann W, Robert N, Pienkowski T, Martin M, Press M, et al; Breast Cancer International Research Group. Adjuvant trastuzumab in HER2-positive breast cancer. $N$ Engl J Med 2011; 365: 1273-1283.

19. Slamon DJ, Godolphin W, Jones LA, Holt JA, Wong SG, Keith $\mathrm{DE}$, et al. Studies of the HER-2/neu proto-oncogene in human breast and ovarian cancer. Science 1989; 244: 707-712.

20. Seidman A, Hudis C, Pierri MK, Shak S, Paton V, Ashby M, et al. Cardiac dysfunction in the trastuzumab clinical trials experience. $J$ Clin Oncol 2002; 20: 1215-1221.

21. Subar M, Lin W, Chen W, Pittman DG. Lack of uniformity in cardiac assessment during trastuzumab therapy. Breast $J$ 2011; 17: $383-390$.

22. Dang CT, Yu AF, Jones LW, Liu J, Steingart RM, Argolo DF, et al. Cardiac surveillance guidelines for trastuzumab-containing therapy in early-stage breast cancer: Getting to the heart of the matter. J Clin Oncol 2016; 34: 1030-1033.

23. Plana JC, Galderisi M, Barac A, Ewer MS, Ky B, Scherrer- 
Crosbie M, et al. Expert consensus for multimodality imaging evaluation of adult patients during and after cancer therapy: A report from the American Society of Echocardiography and the European Association of Cardiovascular Imaging. $J$ Am Soc Echocardiogr 2014; 27: 911-939.

24. Moilanen T, Jokimaki A, Tenhunen O, Koivunen JP. Trastuzumab-induced cardiotoxicity and its risk factors in real-world setting of breast cancer patients. J Cancer Res Clin Oncol 2018; 144: $1613-1621$.

25. Steingart RM, Chandrashekhar Y, Marwick TH. Imaging in cardio-oncology: Where are we and where should we be going? JACC Cardiovasc Imaging 2018; 11: 1209-1211.

26. Liu J, Banchs J, Mousavi N, Plana JC, Scherrer-Crosbie M, Thavendiranathan $\mathrm{P}$, et al. Contemporary role of echocardiography for clinical decision making in patients during and after cancer therapy. JACC Cardiovasc Imaging 2018; 11: 1122-1131.

27. Ishihara M, Mukai H, Nagai S, Mukohara T. Cardiac safety of trastuzumab as adjuvant treatment for Japanese patients with early breast cancer. Int J Clin Oncol 2009; 14: 431 - 435.

28. Sawaki M, Mukai H, Tokudome N, Nakayama T, Taira N, Mizuno T, et al. Safety of adjuvant trastuzumab for HER2-overexpressing elderly breast cancer patients: A multicenter cohort study. Breast Cancer 2012; 19: 253-258.

29. Chien HC, Kao Yang YH, Bai JP. Trastuzumab-related cardiotoxic effects in Taiwanese women: A nationwide cohort study. JAMA Oncol 2016; 2: 1317-1325.

30. Wang SY, Long JB, Hurria A, Owusu C, Steingart RM, Gross CP, et al. Cardiovascular events, early discontinuation of trastuzumab, and their impact on survival. Breast Cancer Res Treat 2014; 146: $411-419$.

31. Leong DP, Cosman T, Alhussein MM, Kumar Tyagi N, Karampatos S, Barron CC, et al. Safety of continuing trastuzumab despite mild cardiotoxicity. JACC CardioOncol 2019; 1: 1-10.

32. Witteles RM. Type I and type II cardiomyopathy classifications are complete nonsense: PRO. https://www.acc.org/latest-in-car diology/articles/2018/05/04/08/41/type-i-and-type-ii-cardiomyop athy-classifications-are-complete-nonsense-pro (accessed October 1, 2019).

33. Kenigsberg B, Wellstein A, Barac A. Left ventricular dysfunction in cancer treatment: Is it relevant? JACC Heart Fail 2018; 6: $87-95$.

34. Guha A, Armanious M, Fradley MG. Update on cardio-oncology: Novel cancer therapeutics and associated cardiotoxicities. Trends Cardiovasc Med 2019; 29: 29-39.

35. Tanaka S, Ikari A, Nitta T, Horiuchi T. Long-term irreversible trastuzumab-induced cardiotoxicity for metastatic breast cancer in a patient without cardiac risk factors. Oxf Med Case Rep 2017; 2017: omx 038
36. Barish R, Lynce F, Unger K, Barac A. Management of cardiovascular disease in women with breast cancer. Circulation 2019; 139: $1110-1120$.

37. Koene RJ, Prizment AE, Blaes A, Konety SH. Shared risk factors in cardiovascular disease and cancer. Circulation 2016; 133: $1104-1114$

38. An J, Sheikh MS. Toxicology of trastuzumab: An insight into mechanisms of cardiotoxicity. Curr Cancer Drug Targets 2019; 19: $400-407$.

39. Kimmick G, Dent S, Klem I. Risk of cardiomyopathy in breast cancer: How can we attenuate the risk of heart failure from anthracyclines and anti-HER2 therapies? Curr Treat Options Cardiovasc Med 2019; 21: 30.

40. Levis BE, Binkley PF, Shapiro CL. Cardiotoxic effects of anthracycline-based therapy: What is the evidence and what are the potential harms? Lancet Oncol 2017; 18: e445-e456.

41. Yeh ET, Chang HM. Oncocardiology: Past, present, and future: A review. JAMA Cardiol 2016; 1: 1066-1072.

42. Lee Chuy K, Nahhas O, Dominic P, Lopez C, Tonorezos E, Sidlow R, et al. Cardiovascular complications associated with mediastinal radiation. Curr Treat Options Cardiovasc Med 2019; 21: 31 .

43. Campia U, Moslehi JJ, Amiri-Kordestani L, Barac A, Beckman JA, Chism DD, et al. Cardio-oncology: Vascular and metabolic perspectives: A scientific statement from the American Heart Association. Circulation 2019; 139: e579-e602.

44. Moslehi J, Fujiwara K, Guzik T. Cardio-oncology: A novel platform for basic and translational cardiovascular investigation driven by clinical need. Cardiovasc Res 2019; 115: 819-823.

45. Lenneman CG, Sawyer DB. Cardio-oncology: An update on cardiotoxicity of cancer-related treatment. Circ Res 2016; 118: $1008-1020$.

46. Lancellotti P, Suter TM, Lopez-Fernandez T, Galderisi M, Lyon AR, Van der Meer P, et al. Cardio-oncology services: Rationale, organization, and implementation. Eur Heart J 2019; 40: $1756-$ 1763.

47. Negishi T, Thavendiranathan P, Negishi K, Marwick TH; SUCCOUR investigators. Rationale and design of the Strain Surveillance of Chemotherapy for Improving Cardiovascular Outcomes: The SUCCOUR Trial. JACC Cardiovasc Imaging 2018; 11: $1098-1105$

48. Zardavas D, Suter TM, Van Veldhuisen DJ, Steinseifer J, Noe J, Lauer S, et al. Role of troponins I and T and N-terminal prohormone of brain natriuretic peptide in monitoring cardiac safety of patients with early-stage human epidermal growth factor receptor 2-positive breast cancer receiving trastuzumab: A herceptin adjuvant study cardiac marker substudy. J Clin Oncol 2017; 35: $878-884$. 